J. Urol. Urogynäkol. AT 2020 $27: 70-74$ https://doi.org/10.1007/s41972-020-00104-8 Online publiziert: 27. März 2020

(c) Der/die Autor(en) 2020

A. Gulacsi · G. Kiss - J. Stühmeier · M. Pedrini · W. Horninger · P. Rehder

Universitätsklinik für Urologie, Medizinische Universität Innsbruck, Innsbruck, Österreich

\title{
Zurückgewonnene Selbstständigkeit bei hoher und kompletter Querschnittslähmung der Frau
}

\section{Zusammenfassung aus urologischer Sicht}

\section{Grundlagen}

In Österreich treten jedes Jahr 200-250 neue posttraumatische Querschnittslähmungen auf [1]. Bei der Diagnose wird die Läsionshöhe mit dem letzten intakten Segment definiert. Dieses wird nach klinischer Untersuchung - Statuserhebung anhand der Dermatome und Kennmuskeln - bzw. mit Hilfe der Bildgebung beschrieben.

Was genau eine hohe Querschnittslähmung ist, wird dabei nicht exakt definiert. Allgemein sind Ausfälle an den oberen Extremitäten ab einem Verletzungsniveau von $\mathrm{C}$ (Cervical)7 und höher zu erwarten. Aus neurologischer und urologischer Sicht wird bereits die Höhe Th(Thoracal)6, wegen der Gefahr des Auftretens einer möglichen autonomen Dysreflexie, die die Harnblase und die Kreislaufregulation betrifft, als wichtige Grenze gedeutet. In der Unfallchirurgie und Intensivmedizin gilt bereits eine Läsionshöhe $\mathrm{C} 2 / 3$ wegen möglicher Ausfälle des Nervus phrenicus als vital kritisch. Aus Sicht der Rehabilitationsmedizin steht die Wiederherstellung der Selbstständigkeit mitunter an oberster Stelle. Diese Darstellung zeigt, dass Verletzungen mit Tetraplegie besondere Komplexität aufweisen [2].

\section{Urologische Akutversorgung und Therapie bei hoher Querschnittlähmung}

Aus urologischer Sicht sind in der Akutphase sofortige Maßnahmen notwendig - die wichtigste stellt dabei die Sicherstellung der Blasenentleerung dar. Diese soll baldmöglichst noch am Unfallort, jedoch spätestens im Schockraum erfolgen, da bei einem spinalen Schock mit einer Harnverhaltung zu rechnen ist. Erst nach der Akutphase ist eine notwendige Abklärung des Blasenlähmungstyps möglich. Nach erfolgter neurologischer und neurourologischer Diagnostik ist es möglich, ein passendes Therapiekonzept zu erstellen [2].

Die Nachsorge weist ebenso eine hohe Komplexität auf, denn regelmäßige neurologische, orthopädische und neurourologische Kontrollen sind unausweichlich. Außerdem werden operative Eingriffe je nach Grad vorgenommen. Es wird auch deutlich darauf hingewiesen, dass im Laufe der Nachsorge auch Konzeptänderungen bzw. Anpassungen vorgenommen werden, da sich die Mobilität und selbst das neurologische Niveau ändern können.

Bei einer hohen Querschnittslähmung verursachen die vegetativen Symptome oft mehr Beschwerden als die des Bewegungsapparates selbst. Nach Angaben von Patienten stellen Blasenund Mastdarmentleerungsstörungen eine belastende Einschränkung im Alltag dar.
Bei suprasakral spinalen Läsionen, wie bei der hohen, kompletten Querschnittslähmung im Besonderen, besteht eine phasische, neurogene Detrusorüberaktivität in Kombination mit einer DetrusorSphinkter-Dyssynergie (DSD) und Asensitivität der Harnblase. Diese vielleicht etwas umständliche, aber umso genauere Bezeichnung für diese Form der neurogenen Harnblase entspricht der geltenden Terminologie der ICS („International Continent Society") und ersetzt Bezeichnungen wie spastische Blase, automatische Blase, Hyperreflexie oder Reflexblase (• Abb. 1).

Das neurourologische Therapieziel muss immer die Protektion des oberen Harntraktes und die Sicherstellung der regelmäßigen Blasenentleerung sein. Des Weiteren gelten das Streben nach höchstmöglicher Selbstständigkeit und die Vermeidung von Harninkontinenz wie auch Harnwegsinfekten als oberste Priorität. Bei tetraplegischen Patienten sind mehrere Maßnahmen zugleich erforderlich, wobei neben der Berücksichtigung der geschlechterspezifischen Unterschiede, je nach vorhandener Funktionstüchtigkeit der Hände, 2 Gruppen unterschieden werden müssen:

- Ausreichend vorhandene oder durch Rehabilitation mindestens $z$. T. wiedergewonnene Handfunktion: In dieser Untergruppe sind beinahe die gleichen Optionen bei Frauen und Männern möglich. Die beste Blasenentleerungsmethode ist der intermittierende Selbstkathe- 


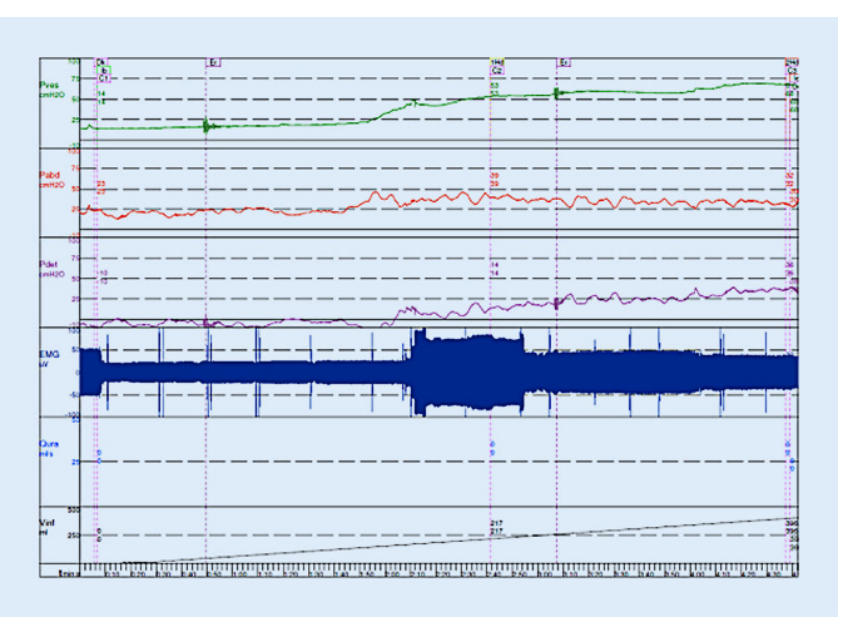

Abb. 1 ॥ Urodynamik, Detrusorüberaktivität, DSD (Detrusor-Sphinkter-Dyssynergie)

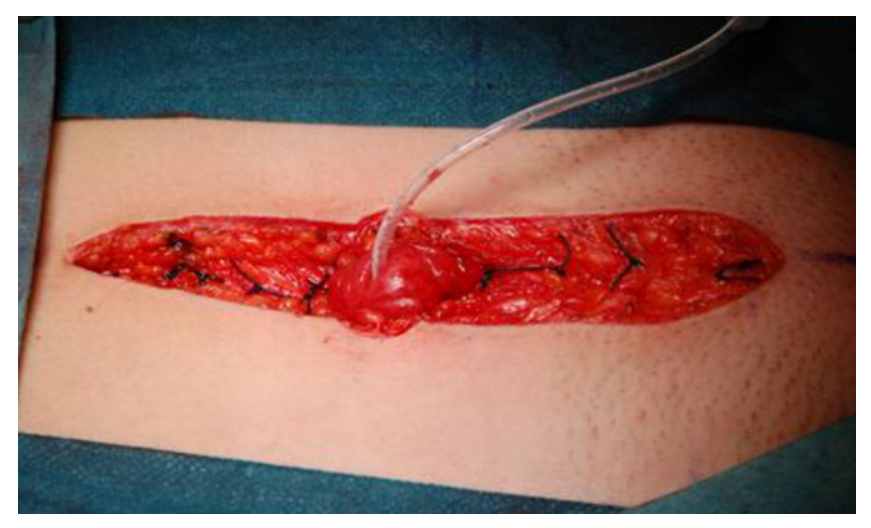

Abb. $3<$ Mitrofanoff-Stoma

terismus, ggf. in Kombination mit einer antimuskarinergen Therapie. Es zeigt sich aber bereits hier, dass der Katheterismus generell und der Selbstkatheterismus im Speziellen für Frauen, die auf einen Rollstuhl angewiesen sind, wesentlich umständlicher ist als für Männer. Des Weiteren muss in Betracht gezogen werden, dass die medikamentöse Unterdrückung der Detrusorüberaktivität oft keine $100 \%$ ige Kontinenz sichert und v. a. bei Harnwegsinfektionen ein Urinverlust auftreten kann. Dagegen können sich männliche Betroffene durch die Anwendung von Kondomurinalen besser schützen als Frauen, die Einlagen verwenden. Als letztes Mittel in solchen Fällen kann die operative Vergrößerung der neurogen beeinträchtigen Speicherkapazität mit diversen Methoden der Blasenaugmentation, bei beiden Geschlechtern, Abhilfe schaffen.

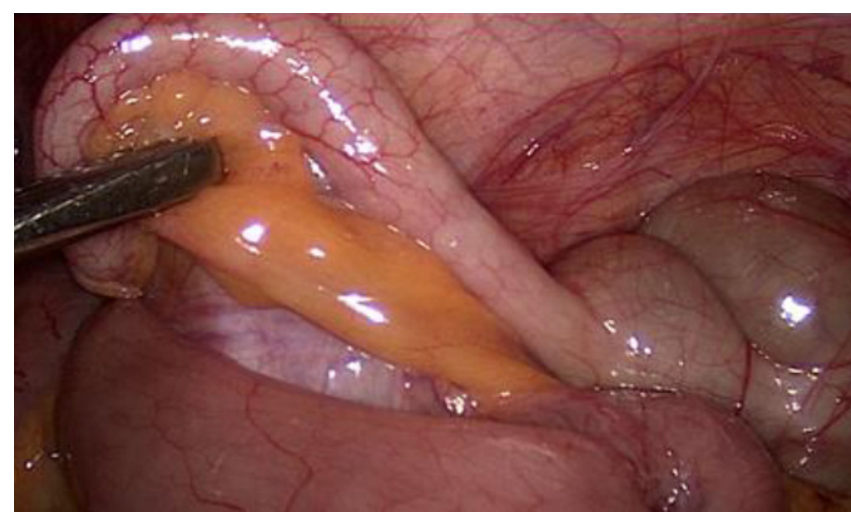

Abb. 2 ム Laparoskopische Abklärung

bestehen, die im Folgenden dargestellt werden:

1. Der Selbstkatheterismus kann ohne ausreichende Funktionstüchtigkeit der Hände, im speziellen des Pinzettengriffs, weder im Rollstuhl noch im Bett durchgeführt werden.

2. Selbst der Fremdkatheterismus geht oft mit Hindernissen einher, der durch die notwendige Lagerung und/ oder Transfer erschwert wird. Das bedeutet, dass die Betroffenen für die richtige Lagerung und für den Katheterismus auf Hilfe weiterer Personen angewiesen sind. Dies ist 4- bis 5-mal täglich sicherzustellen und bedeutet einen höheren Pflegeaufwand wie die hochgradige Einschränkung der Selbstständigkeit der Betroffenen.

3. Auch die Schwierigkeiten bei der Versorgung der Harninkontinenz mit ihren Sekundärgefahren, wie dermatologische Komplikationen oder Harnwegsinfekte, müssen berücksichtigt werden.

\section{Fallbeispiele aus der Universitätsklinik Innsbruck}

Für Betroffene mit einer hohen Querschnittslähmung rückt die allgemeine Selbstständigkeit auch hinsichtlich urologischer Zielsetzungen nach vorne. Dies bedeutet das Erlernen einer Blasenentleerungsmethode, die ohne fremde Hilfe auskommt, auf jeder Toilette durchführbar ist und ein hohes Maß an Sicherheit vor Harninkontinenz gewährleistet. Anhand der vorliegenden Fallbeispiele wird der Verlauf der Therapie zweier jun- 
ger Patientinnen beschrieben, die nach Abschluss ihrer Therapie wieder in ihren beruflichen Alltag integriert werden konnten. Diesen, im Vorfeld formulierten Zielen entsprechend, wählten die behandelnden Urologen die Therapieoption des katheterisierbaren Stomas. Oberstes Ziel war es, durch die Konfiguration eines Mitrofanoff-Stomas (Appendikovesikostomie) am Unterbauch die Selbstkatheterisierung im Rollstuhl ohne Fremdhilfe zu ermöglichen. Für diese Option mussten die ausreichende Speicherfunktion der Harnblase sowie die weitgehende Symptomfreiheit in Zusammenhang mit einer autonomen Dysregulation gesichert sein. Diese Voraussetzungen müssen präoperativ urodynamisch unter der Wirkung von Antimuskarinika nachgewiesen werden.

In den letzten 5 Jahren wurden an der Universitätsklinik Innsbruck 2 Sportlerinnen im Alter von 22 und 27 Jahren mit posttraumatischer Tetraplegie betreut, deren Therapieverlauf im Folgenden dargestellt wird.

Die Patientinnen erlitten aufgrund von Sportunfällen beinahe idente Verletzungen mit Luxationsfraktur des 5. bzw. 6. Halswirbelkörpers, mit konsekutiver, kompletter Querschnittslähmung ab der neurologischen Höhe C6 und inkompletten Ausfällen ab der Höhe C4/5. Bei dem Grad ihrer Verletzungen blieb die Innervation ab C5 aufwärts z. T. intakt. Dies bedeutet im Allgemeinen, dass der N. medianus (C5 Th2) teilweise funktionstüchtig bleibt und dadurch grobmotorische Bewegungsabläufe der Hände möglich sind. Durch eine Extension des Handgelenks kommt es zur Greifbewegung zwischen dem Daumen und den restlichen Fingern. Somit kann ein Einmalkatheter gegriffen und selbstständig gesetzt werden.

Dies muss im Rollstuhl sitzend erfolgen, da die ausgehende Kraft der Armmuskulatur bei diesen Patientinnen nicht ausreicht, um sich selbstständig aus dem Rollstuhl zu heben, um sich einen konventionellen Einmalkatheter zu setzen. Des Weiteren wurden im Vorfeld in beiden Fällen durch entsprechende medikamentöse Therapie eine ausreichende Blasenkapazität und die motorische Um-

J. Urol. Urogynäkol. AT 2020 · 27:70-74 https://doi.org/10.1007/s41972-020-00104-8

(c) Der/die Autor(en) 2020

\section{A. Gulacsi · G. Kiss · J. Stühmeier · M. Pedrini · W. Horninger · P. Rehder}

\section{Zurückgewonnene Selbstständigkeit bei hoher und kompletter Querschnittslähmung der Frau. Zusammenfassung aus urologischer Sicht}

\begin{abstract}
Zusammenfassung
Statistisch gesehen sind weltweit mehr als 7 Mio. Menschen von einer Rückenmarkverletzung, darunter 2,7 Mio. von einer manifesten Querschnittslähmung, betroffen. In Österreich sind etwa 50.000 Patienten ausschließlich durch die Hilfe eines Rollstuhls mobil, davon sind etwa 4000 von einer Querschnittslähmung betroffen. Die Betreuung dieser Patienten stellt in der Praxis oft eine besondere Herausforderung dar. Große Bedeutung wird der Nachsorge beigemessen, die aufgrund spezieller Begleitumstände und der Komplikationsgefahren immanent wichtig ist. Aus urologischer Sicht ist eine
\end{abstract}

neurourologische Betreuung unerlässlich. Dabei verändern sich die Therapieoptionen nach dem Maß des Verletzungsniveaus und sind zugleich geschlechterspezifisch. In diesem Artikel wird über die urologischen Aspekte der Versorgung von Frauen diskutiert, die von einer hohen Querschnittslähmung (Tetraplegie) betroffen sind.

\section{Schlüsselwörter}

Tetraplegie · Blasenfunktionsstörung . Mitrofanoff-Stoma - Selbstkatheterismus . Pinzettengriff

\section{Regained independence in high and complete paraplegia in women. A summary from the urological perspective}

\section{Abstract}

From a statistical point of view, more than 7 million people worldwide are affected by spinal cord injuries, 2.7 million of these by manifest paraplegia. In Austria, approximately 50,000 patients rely exclusively on a wheelchair for mobility, with around 4000 of these affected by para or tetraplegia. The care of these patients is often particularly challenging in daily practice. Great significance is attached to aftercare, which is eminently important due to the special concomitant circumstances and the risk of complications. From a urological perspective neuro-urological care is essential, with treatment options changing according to the extent and level of injury and being genderspecific. This article discusses the urological aspects of the treatment of women with high paraplegia (tetraplegia).

\section{Keywords}

Tetraplegia · Bladder dysfunction · Mitrofanoff stoma $\cdot$ Self catheterisation $\cdot$ Pinch grip setzung des Selbstkatheterismus über ein Nabelstoma gesichert.

Bei den Patientinnen wurde unter antimuskarinerger Therapie eine Blasenkapazität von $400-600 \mathrm{ml}$ erreicht. Durch die 4- bis 6-malige Anwendung des Fremdkatheterismus am Tag konnte eine dauerhafte Kontinenz erreicht werden.

\section{Operative Vorgangsweise be oben genannten Fällen}

Als erster operativer Schritt wird eine laparoskopische Abklärung durchgeführt, um den Appendix zu identifizieren und zu prüfen. Der Zustand des Appendix ließ in beiden Fällen die Stomakonfiguration zu. Über einen Unterbauchschnitt wurde die vordere Blase extraperitoneal exploriert und der in diesen Fällen gut durchblutete Appendix intraperitoneal mobilisiert. Ein katheterisierbares, antirefluxives Stoma wurde nach Mitrofanoff konfiguriert. Es traten keine intraoperativen Komplikationen auf (• Abb. 2 und 3 ).

Im Zusammenhang mit dem Mitrofanoff-Stoma entstehen die häufigsten Komplikationen durch die Verengung und die Vernarbung des Stomas. Um diese zu vermeiden, wurde ein Dauerkatheter zur Schienung für 6-8 Wochen (abhängig vom postoperativen Verlauf) 

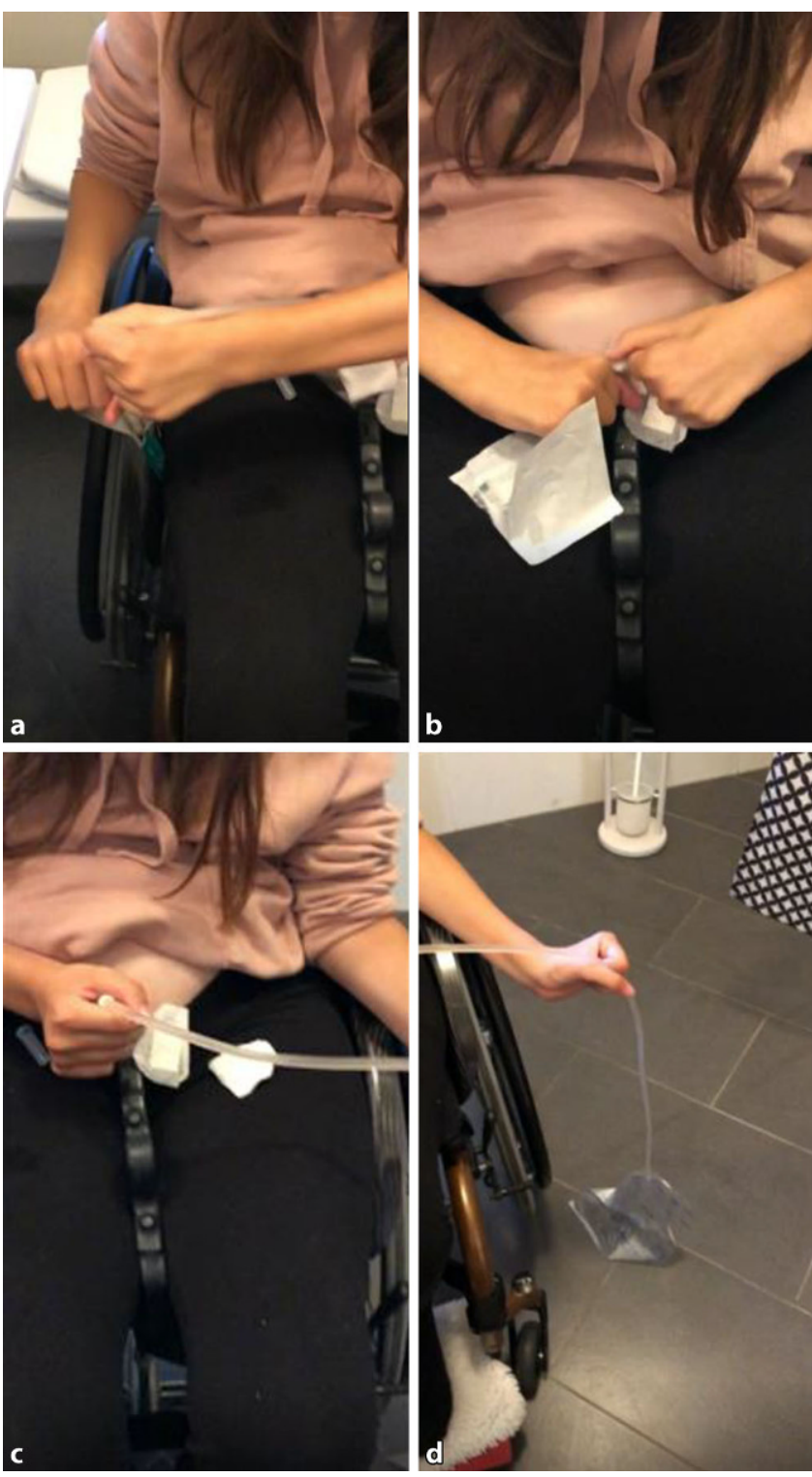

eingesetzt, damit die Patientinnen in der Folge mit dem Selbstkatheterismus anfangen konnten. Die postoperativen Kontrollen zeigten in beiden Fällen zufriedenstellende urodynamische $\mathrm{Be}$ funde. Im Konkreten bedeutete das, dass die Blasenkapazität beibehalten werden konnte und die Patientinnen durch die Einnahme von Antimuskarinika weiterhin kontinent blieben.

\section{Die schrittweise Methode des Selbstkatheterismus}

1. Positionierung und Vorbereitung im Rollstuhl

2. Einhaken eines Bügels zum Aufspannen der Hose
Abb. $4<$ Selbstkatheterismus im Rollstuhl

\section{Fazit}

Beide Patientinnen berichteten über eine neu gewonnene, sehr erfreuliche Selbstständigkeit. Zuvor waren beide Patientinnen bei der Blasenentleerung auf mindestens 2 Personen angewiesen. Sie mussten aus dem Rollstuhl gehoben und für den Katheterismus mit gespreizten Beinen positioniert und wegen ihrer Spastizität festgehalten werden.

Die neu gewonnene Selbstständigkeit war für beide Patientinnen ein entscheidender Schritt in der Rehabilitation zurück in eine autonome Gestaltung des Alltags.

Unabhängig von den positiven Rehabilitationserfolgen aus Sicht der Patienten kann man feststellen, dass dieses operative Verfahren zum einen sehr ressourcenschonend ist und des Weiteren sowohl für die Patienten und das Pflegepersonal aufgrund der leichteren Handhabe eine enorme Erleichterung bzw. Entlastung auf mehreren Ebenen darstellt.

\section{Korrespondenzadresse}

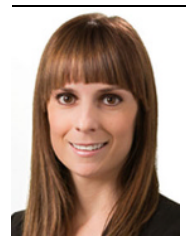

Dr. A. Gulacsi, FEBU

Universitätsklinik für Urologie, Medizinische Universität Innsbruck

Anichstraße 35, 6020 Inns-

bruck, Österreich

Alexandra.gulacsi@ tirol-kliniken.at

J.Stühmeier
Universitätsklinik für Urologie,
Medizinische Universität
Innsbruck
Anichstraße 35,6020 Inns-
bruck, Österreich
jannik.stuehmeier@
i-med.ac.at

Funding. Open access funding provided by University of Innsbruck and Medical University of Innsbruck.

\section{Einhaltung ethischer Richtlinien} dem Harn im Schlauch fließt, den Harnbeutel loslassen). Der Sog im Schlauch sorgt für eine komplette Blasenentleerung (• Abb. 4d).

7. Als letzter Schritt werden alle Einmalprodukte entsorgt.
Interessenkonflikt. A. Gulacsi, G. Kiss, J. Stühmeier, M. Pedrini, W. Horninger und P. Rehder geben an, dass kein Interessenkonflikt besteht.

Für diesen Beitrag wurden von den Autoren keine Studien an Menschen oder Tieren durchgeführt. Für die aufgeführten Studien gelten die jeweils dort angegebenen ethischen Richtlinien. Für Bildmaterial oder anderweitige Angaben innerhalb des Manuskripts, 
über die Patienten zu identifizieren sind, liegt von innen und/oder ihren gesetzlichen Vertretern eine schriftliche Einwilligung vor.

Open Access. Dieser Artikel wird unter der Creative Commons Namensnennung 4.0 International Lizenz veröffentlicht, welche die Nutzung, Vervielfältigung Bearbeitung, Verbreitung und Wiedergabe in jeglichem Medium und Format erlaubt, sofern Sie den/die ursprünglichen Autor(en) und die Quelle ordnungsgemäß nennen, einen Link zur Creative Commons Lizenz beifügen und angeben, ob Änderungen vorgenommen wurden.

Die in diesem Artikel enthaltenen Bilder und sonstiges Drittmaterial unterliegen ebenfalls der genannten Creative Commons Lizenz, sofern sich aus der Abbildungslegende nichts anderes ergibt. Sofern das betreffende Material nicht unter der genannten Creative Commons Lizenz steht und die betreffende Handlung nicht nach gesetzlichen Vorschriften erlaubt ist, ist für die oben aufgeführten Weiterverwendungen des $\mathrm{Ma}$ terials die Einwilligung des jeweiligen Rechteinhabers einzuholen.

Weitere Details zur Lizenz entnehmen Sie bitte der Lizenzinformation auf http://creativecommons.org/ licenses/by/4.0/deed.de.

\section{Literatur}

1. Allgemeine Unfallversicherungsanstalt (AUVA) (2018) Auszug aus der Statistik 2017

2. Blackmer J (2003) Rehabilitation medicine:

1. autonomic dysreflexia. Can Med Assoc J 169:931-935

3. Taweel WA, Seyam R (2015) Neurogenic bladder in spinal cord injury patients. Res Rep Urol 7:85-99

4. Arbeitskreis Neuro-Urologie der Deutschsprachigen, Medizinischen Gesellschaft für Paraplegie (DMGP) S2k-Leitlinie der Deutschsprachigen Medizinischen Gesellschaft für Paraplegie (DMGP) Neuro-urologische Versorgung querschnittgelähmter Patienten. AWMF Register Nr. 179/001

5. Benevento BT, Sipski ML (2002) Neurogenic bladder, neurogenicbowel, and sexual dysfunction in people with spinal cord injury. Phys Ther 82:601-612

6. Gulacsi A, Kiss G, Pedrini M, Stühmeier J, Horninger W, Rehder P (2019) Blasenentleerung bei hoher, kompletter Querschnittlähmung der Frau.Urologik3:6-7. (BUV-ÖGUTagung)

Hinweis des Verlags. Der Verlag bleibt in Hinblick auf geografische Zuordnungen und Gebietsbezeichnungen in veröffentlichten Karten und Institutsadressen neutral.

\section{Termine \& Neues}

\section{Jahrestagung der Medizinischen Kontinenzgesellschaft Österreich} "Der verNETZte Beckenboden"

9.-10. Oktober 2020

LFI Oberösterreich - Seminarhaus auf der Gugl

Auf der Gugl 3, 4021 Linz

Info \& Anmeldung unter www.kontinenzgesellschaft.at

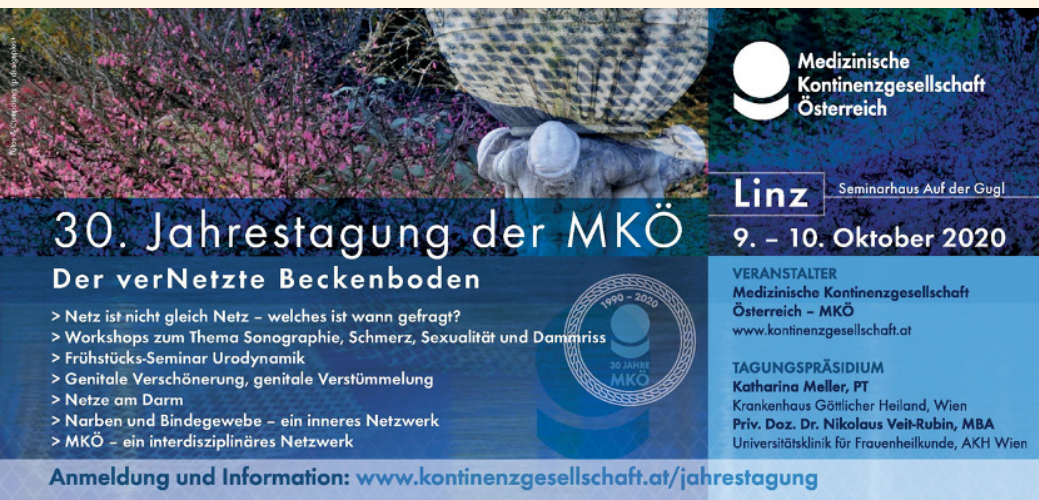

\section{Neu: Fachvorträge im YouTube-Kanal der MKÖ}

Die MKÖ verfügt seit Juni letzten Jahres über einen eigenen YouTube-Kanal. Neu ist die zusätzliche Rubrik „Fachvorträge“. Sie beinhaltet eine Auswahl an Vorträgen im Rahmen der letztjährigen Jahrestagung: www.youtube.com/kontinenz (Playlist „Fachvorträge“).

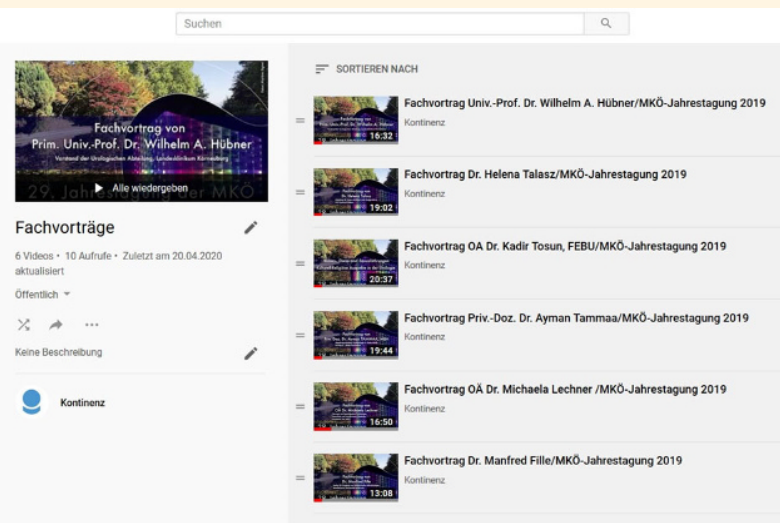

Welt-Kontinenz-Woche: 5 Tage für den Beckenboden

Täglich ein virtueller medizinischer Vortrag und praktische Tipps von Physiotherapeutinnen

Im Rahmen der heurigen Welt-Kontinenz-Woche (15.-19. Juni 2020) widmete sich die MKÖ dem Beckenboden und zeigte, wie das "Work-out für diesen unsichtbaren Muskel“ funktioniert. Dazu gab es umfassende medizinische Information zu unterschiedlichen Fragestellungen rund um Ursachen, Diagnose und Behandlungsmöglichkeiten.

Jeden Tag wurde in Form von virtuellen Vorträgen für Betroffene und Angehörige ein Thema rund um den Beckenboden besprochen. Das Programm bestand aus zwei Teilen: Information durch einen Mediziner/eine Medizinerin mit der Möglichkeit, per Chat anonym Fragen zu stellen, und lebendig präsentierte, praktische Tipps von Physiotherapeutinnen.

Alle Videos stehen nun im YouTube-Kanal der MKÖ zur Verfügung: www.youtube.com/kontinenz 